\title{
WASHING MACHINE CONTROLLER WITH A NEW PROGRAMMING METHOD
}

\author{
Andrzej MILECKI*, Roman REGULSKI* \\ *Poznan University of Technology, ul. Piotrowo 3, 60-965 Poznań, Poland \\ andrzej.milecki@put.poznan.pl, roman.regulski@put.poznan.pl
}

received 10 October 2016, revised 7 December 2017, accepted 11 December 2017

\begin{abstract}
In the paper the newly designed at Poznan University of Technology (PUT) washing machine controller is presented. The commonly used in washing machines sensors, drives and other input-output elements are briefly described. The designed at PUT controller is based on 32-bit STM32 microcontroller. The used in this controller modules are described and their input/output signals and basics of operations are presented. The developed in the controller user-machine communication devices, elements and methods are described. The paper presents new washing machine programming methods and implementation software, such as voice recognition and intelligent programming of washing machine that were applied in the new controller.
\end{abstract}

Key words: Washing Machine, Programming, Communication, Mechatronics

\section{INTRODUCTION}

In the last two decades the expansion of sector producing house equipment in Poland is observed. The production quantities and assortment of produced refrigerators, washing machines, cookers, etc. have increased significantly. Also new devices like dishwashers have been introduced to production. In Poland such big and famous companies like Indesit (Łódź), Whirpool (Wrocław), Electrolux (Świdnica, Żarów), BSH (Łódź, Wrocław), Samsung EPM (Wronki), Amica, Beko, Candy, Gorenje, Indesit have started production factories. Nowadays, Poland is one of the biggest producers of "white goods" in Europe. The export of sector producing house equipment in Poland is about $3 \%$ of the total polish export. In the first quarter of 2016 in Poland about 5.6 million of so-called "big-white-goods" devices have been produced.

Recently a new group of household electronics is strongly developed, which may work in a network called Internet-of-Things. Continuous miniaturization in electronic packages and growth in microcontroller performance causes new concept of an environment where everyday objects are connected to the Internet. This will lead users to consume resources more efficiently (Bourgeois et al., 2014; Risteska Stojkoska and Trivodaliev, 2017).

Due to the growing demand for natural Human Machine Interfaces, a lot of concepts for robotic system that allows users to program an industrial robot using gestures and speech are proposed. Examples using ASR (automatic speech recgniotion) are described by (Anusuya and Katti, 2010; Neto et al., 2010; Norberto Pires, 2005).

In the field of medical systems very futuristic applications can be found. In the paper (Gundogdu et al., 2017) ASR was developed to control the prosthetic robot arms. These solutions can be also applied in Smart Homes such as voice interface for the elderly people (Portet et al., 2013) or for improved comfort and autonomy at home (Chahuara et al., 2017).
Gestures are another natural form of communication between humans. In the robotics field, several works have been done in order to identify and recognize motions and gestures performed by humans (Tsarouchi et al., 2016a; Tsarouchi et al., 2016b).

In 2009 at Poznan University of Technology (PUT) a project financed by polish National Centre of Research and Development was started. Its main goal was to design of a new, advanced washing machine microcontroller. In this project some new solutions have been proposed, tested and implemented in the controller.

In most commonly produced washing machines simple 8-bit microcontrollers are applied. For the last ten years, there are 32bit microcontrollers on the market (Brown, 2012; Mallikarjun, 2006), cost of which compared to the cost of 8-bit microcontrollers, is not significantly higher. However the design of controllers based on 32-bit microcontrollers is much more difficult and time consuming. This applies especially to startup process of 32-bit microcontrollers, which is much more complex. Moreover the available to such microcontrollers library is big. The design of printed circuit board (PCB) for 32-bit microcontroller requires high experience of the designer. The programming, testing and debugging processes are also slightly different and more complicated.

At the beginning of the paper the washing machine description is given. Its basic operation. processes and control methods are shortly described (Milecki and Pittner, 2015). Then the structure of designed at PUT washing machine controller is presented. In this controller a 32-bit STM32 microcontroller is used. In order to achieve flexibility the controller hardware structure is modularbased. The individual modules are shortly described. Basic structure, the modules connection, their communication protocols and basics of cooperation between them are also briefly characterized. Also the used in the controller most important electronic circuits are described. In the next point the applied methods for communication between the user and the controller are presented. The main functions and possibilities of washing machine programming are described. The so called "intelligent" programming method is 
presented. Also the voice communication in washing program settings is described. The most important advantages of a new controller are emphasized.

\section{WASHING MACHINE INPUT AND OUTPUT ELEMENTS}

In the modern washing machines several sensors and actuators are applied (Bascetta et al., 2012). Some sensors can measure the parameters, giving analog electrical signals on the outputs. This enables control of the water level and temperature, spin speed, cycle program, load balancing, child lock systems, and noise reduction systems. For example, a push button switch is used as a "door-closed" sensor, that allows a complete circuit when the closed door pushes on it. Another example is a pressostat called as "fill-level" sensor, which measures the level of the water in a drum. This is in fact a linear pressure sensor, in which there are two chambers separated with elastic membrane. The upper chamber is directly connected to the atmosphere and the lower chamber is connected through a pipe with the bottom of the drum, where the pressure is proportional to the level of the water. As a result the diaphragm bends in proportion to the difference of pressures in both chambers. The membrane moved the connected to it a ferromagnetic core, which is located in the middle of the coil supplied by sinusoidal generator.

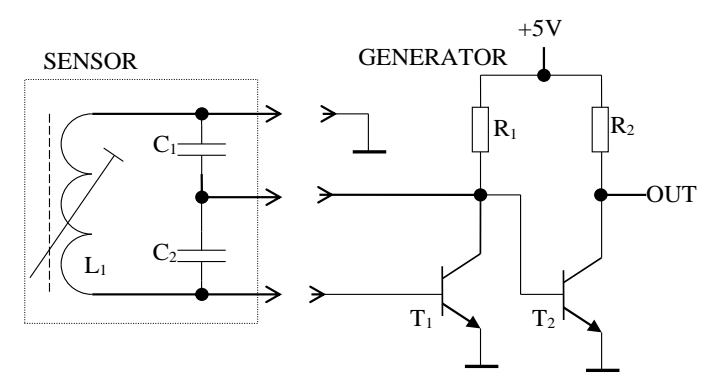

Fig. 1. Pressostat and its electronic circuit

The pressostat electric circuit is shown in Fig. 1. It consists of a NPN transistor, which input (Base) is connected to the coil, which inductance changes by ferromagnetic core according to the level of the water. The coil is connected to two capacitors, which creates a resonance circuit. This circuit creates a Colpitts generator, which frequency changes proportionally to the coil inductance changes. The output signal from generator is given to analog input of the microcontroller. If there is no water in a drum the generated frequency is equal to $26.7 \mathrm{kHz}$ and when the drum is fully filled its frequency is equal to $20.8 \mathrm{kHz}$.

Nowadays in most washing machines, for temperature measurement the NTC thermistor is used. This is a semiconductor element with a non-linear resistanc-temperature characteristic (Fig. 2). This characteristic may be approximated by the following equation:

$T=-28.8 \ln (R)+294.3$

where: $T$ - temperature in ${ }^{\circ} \mathrm{C}, R-\mathrm{NTC}$ thermistor resistance in Ohms.

In washing machine controller designed at PUT, this curve is reversed as shown in Fig. 2, stored in microcontroller's memory and used for linearization.

In cheap washing machines single phase brash AC motor is used, which typically is supplied by 230 VAC. The velocity of this motor is controlled by changing the supply voltage. Usually to this end a simple triac is used, which is switched on by pulses generated by the controller.

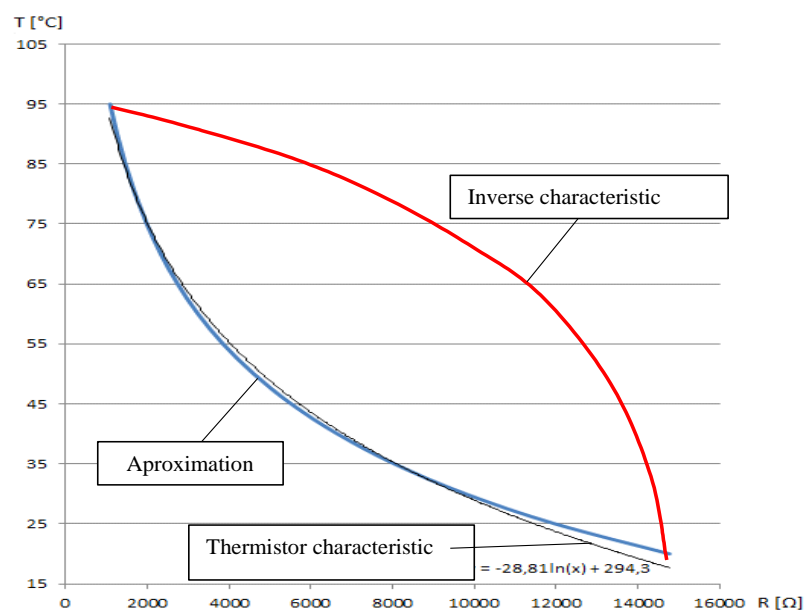

Fig. 2. NTC thermostat characteristic, its approximation (blue) and inverse curves (red)

Nowadays in advanced modern washing machines the mentioned above single phase $A C$ motor is replaced by special inductive motor or by a multi poles brushless DC motor, which construction is similar to torque motor. In this motor there are several coils on a stator and permanent magnets mounted in a rotor. The position of the rotor is measured by Hall sensors, which output signals are used to control of coils switching. These type of motors are called "direct drive" and can rotate with low velocity. Thanks to this the application of a belt transmission is not necessary. For control of this motor a specialized and complicated controller should be applied.

Other output elements used in washing machines are: water pump, electro-valves, heater and door lock electromagnet. Typically these elements are switched on and off by relays or by triacs. In some washing machines also MEMS acceleration sensors are used, which enable the measurement of drum oscillations. Thanks to this the balance of laundry in the drum is controlled and the rotational velocity is adjusted accordingly, assuring safe and silent work. In some washing machines following sensors are used: leak detector, spin out-of-balance detector and mains failure sensor. They generate only on/off type signal, which is connected to the digital inputs of the controller. The heater is also controlled by triac, which is a very durable low-cost semiconductor element. Furthermore triacs are used for switching on and off of the electro valves and pump motor. In many washing machines produced nowadays, a commutated one-phase AC motor is applied, velocity of which is controlled using a triac in a wide range.

\section{STRUCTURE OF 32-BIT WASHING MACHINE CONTROLLER}

Since 1980 the microcontrollers are used as the washing machine controllers. This enabled the application of LED or LCD displays and creation of some buttons for programming and washing process supervision. Thanks to the application of microprocessors the washing process can be controlled much more accu- 
rately. Thanks to advanced control algorithms applied in microcontrollers, washers use less electricity to run the machine and also to set the water to the right temperature levels. The scheme block diagram of a new washing machine controller, which is designed at Poznan University of Technology is presented in Fig. 3. It is based on 32-bit microprocessor.

In order to achieve flexibility the modular structure of the controller was proposed. The basis is main board, on which the CPU board, input sensor block, communication ports like RS485 and USB, EEPROM and buzzer module were implemented. The heart of the controller is CPU board which is connected to the mainboard with a slot, enabling its easy replacement. In the CPU board the microcontroller type STM32F103ZET6 is installed. It is a 32-bit microcontroller based on CortexM3 core. The main board may communicate with different users interfaces. The simplest is the on/off switching panel and the most advanced is a color touchpanel. The next controller board was high power unit, which is responsible for constant velocity control of the drum motor, and for switching on/off of such elements like: heater, valves, pump, door lock electromagnet etc.

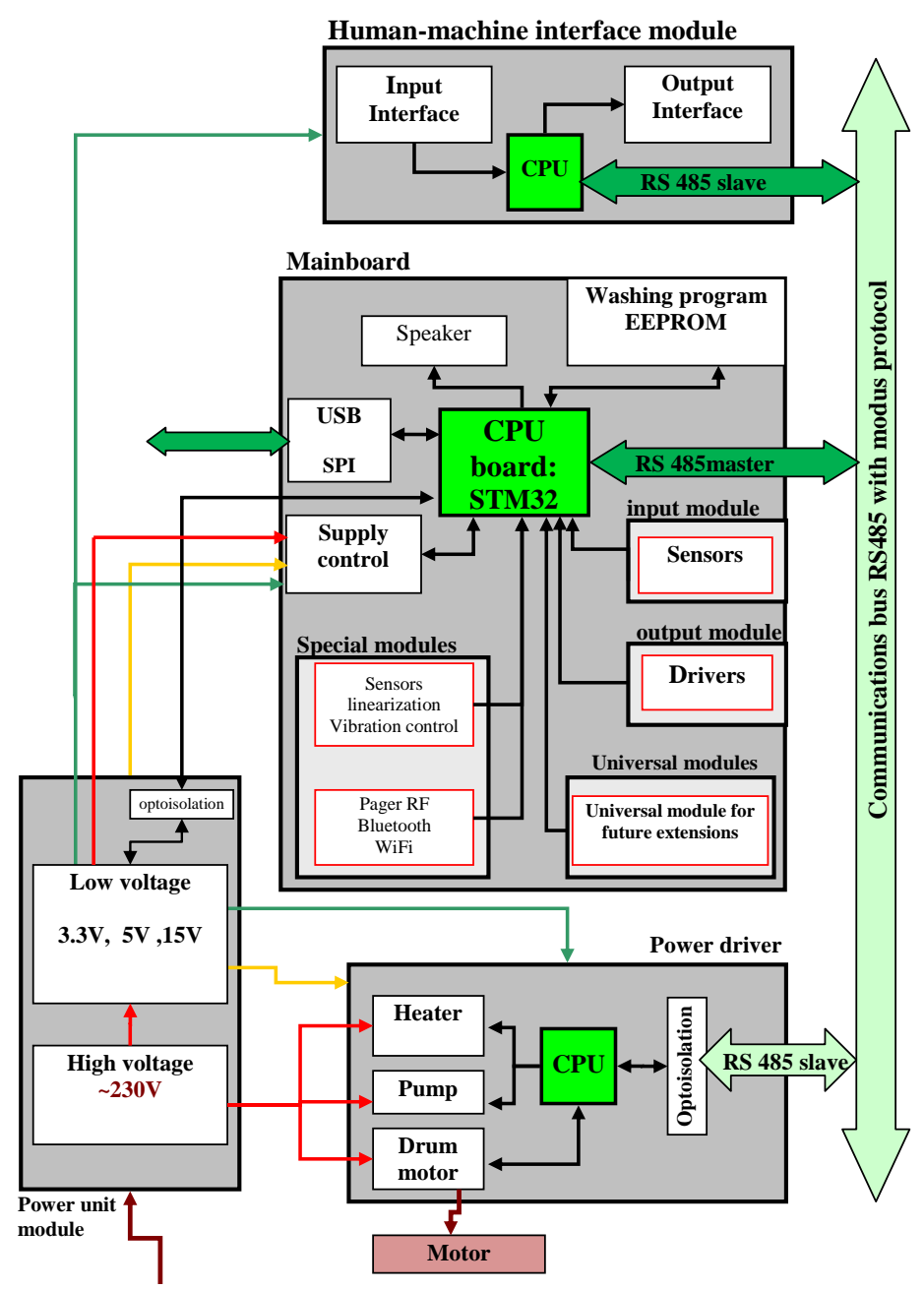

Fig. 3. Washing machine controller block scheme

All modules communicate with each other using serial interface network. In the developed solution, different single electronic modules can be used. Thanks to this the controller may be easy adopted to different requirements, which is an important advantage. Each module may be autonomous, individually programmed unit assuring more safety work. In this approach, the user may design and create the controller's structure, its capacity and ability and finally set its price. Moreover, the same main controller may be used in different washing machines but with different types of additional modules, for example drive controller or user interfaces. Thanks to such solution, the testing and in particular certification process is facilitated. In the designed prototype the digital outputs are connected through relays or through linear amplifier type ULN2003A. All digital inputs and outputs are opto-isolated.

\section{COMMUNICATION BEETWEEN MODULES IN THE WASHING MACHINE CONTROLLER}

In the described here washing machine controller three serial busses were implemented: SPI, RS-485 and USB, which assured internal communication between modules. The SPI was used for programming purposes, testing and commissioning, debugging and communication with EEPROM. The RS-485 was used for communication with High Power Controller like drive controller, relay modules, with user interfaces (LCD) and with Wireless Interfaces Controller. Throughout the project a few human-washing machine interfaces were designed, built and tested. The first one was a mechanical one with typical to washing machines rotary switch and LCD display. The last one was a color touch-screen 
(Fig. 4). The controller was also equipped with a voice interface module which could be used for communication with, for example, blind users. The last interface was a USB, which enabled the wire communication with a PC based computer and was used for testing and diagnosis of the washing machine. In the project our proprietary direct drive controller was designed and built, but almost every other washing machine drive system could be used, i.e. single phase serial motor with triac, direct drive or synchronous PM motor.

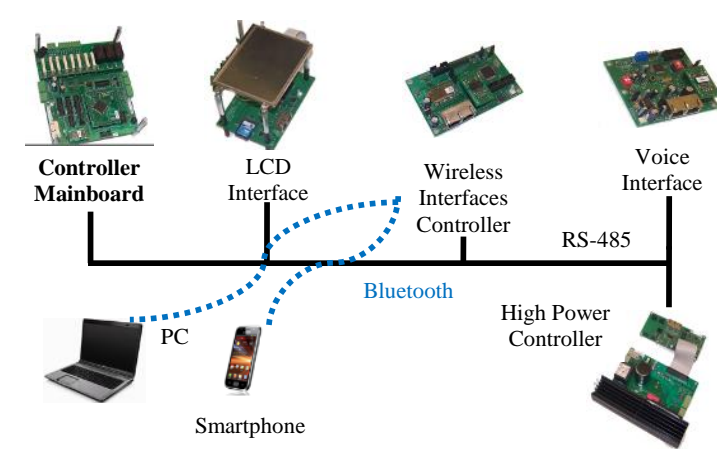

Fig. 4. View of the controller communication RS-485 interface

Additionally the wireless communication card was designed and connected to the controller. It enabled the connection with any device using Bluetooth system, for example with smartphone, PC, or pager. The special software for smartphone (Samsung S5) and PC (laptop) was written and implemented. It enabled the programming and supervision of washing machine using standard programs or user-customized ones. Smartphone or PC may be used instead of typical washing machine interface. Moreover, a so-called by us "intelligent programming" method was proposed and implemented. It enabled settling of the washing parameters using questions and answers. This method utilized control methodology based on fuzzy logic. The next interface was a USB, which enabled the cable communication with PC. Additionally a special software for the service purposes thus for diagnostic, was worked at PUT. It could be activated and all washing machine elements could be tested in the diagnostic mode. Such solution is very useful and enable fast testing and washing machine diagnosis.

\section{VOICE COMMUNICATION AND INTELLIGENT WASHING MACHINE PROGRAMING}

The new possibilities to connect washing machine controller with PC or smartphone provide further flexibility and enhanced user experience. The voice communication enables the intuitive programming of a washing machine. In the designed controller the Google Speech Recognition System was adapted and used. This system is easy to use and assures the acceptable quality of voice recognition.

The software for PC was written in Microsoft Visual Studio 2010 environment, using object programming language C\#. A start/stop algorithm was used for voice recognition, which enabled the recognition of word starting and ending points. The voice signal from the microphone was constantly analyzed using RMS values calculation. For every ongoing $20 \mathrm{~ms}$ frame, the RMS value was calculated and compared with the previous one. If the set up threshold was exceeded, the starting or ending point of word would be detected. In the algorithm additional security tools like: minimum and maximum saying time were used. The command saying time and silence time were set basing on average value of energy, calculated as voice signal RMS during the time $200 \mathrm{~ms}$. These rules were implemented into an algorithm, which eliminated the false recognition of short brakes between words, which may be taken by the algorithm as stop. The scheme of the described above algorithm is shown in Fig. 5. The same algorithm was implemented on a smartphone with Android operating system, but in this case the program was written in Java.

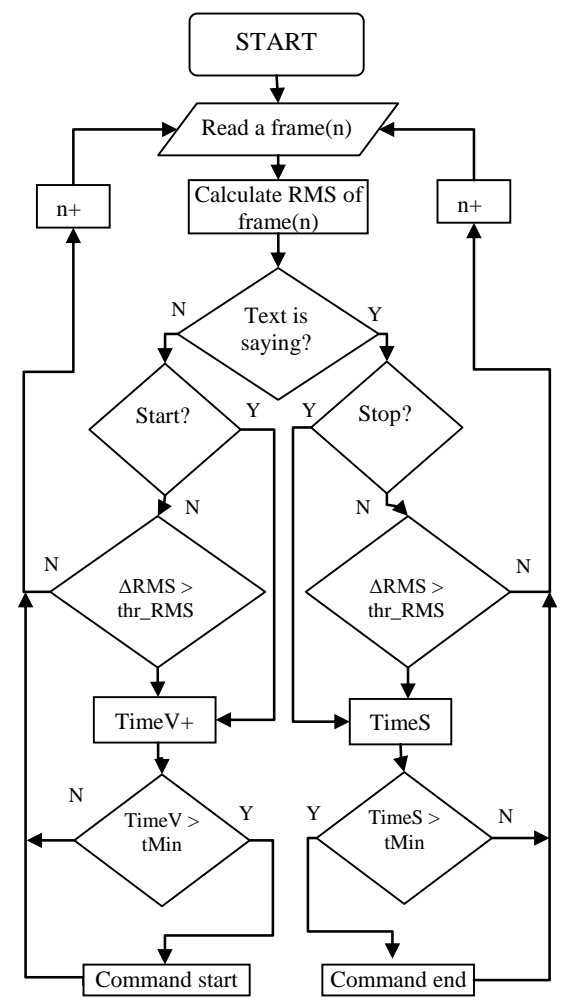

Fig. 5. Start/stop word recognition algorithm scheme

In order to check the efficiency of the voice recognition, the system was tested. In the investigations 5 people were involved. All of them used the same 6 words i.e. commands, repeated 20 times. In these tests following parameters were considered (see Tab. 1): server response time, confidence of recognition and recognition efficiency, defined as a proportion of properly recognized command to saying commands.

The investigations have shown that the recognition efficiency was almost $90 \%$ and the server response time was about $1.3 \mathrm{~s}$, which is quite acceptable.

For the designed washing machine controller a few new programming methods were implemented. The first one is "Classic", which enabled to choose the ready to use, useful washing programs. This programs are commonly prepared by the producers, like: Synthetics, Cottons, Wool, Delicate, Sensitive, Quick and so on. The user may only chose one of them using mouse when PC is applied or pressing the touch-screen when smartphone is connected. There is also a possibility to change parameters using keyboard.

The second method utilizes the advantages of voice communication. In this case the user can "tell" the controller the name of the program and the values of parameters. For example, the user may only tell: "cotton, temperature 60 , rotation speed 1200 , dirt medium, rinsing 2 , delay 60 ". The controller repeats every 
word after recognition and the user has the opportunity to improve it repeating the last mentioned word.

Tab. 1. Speech recognition investigation results

\begin{tabular}{|c|c|c|c|}
\hline User & $\begin{array}{c}\text { Response } \\
\text { time [ms] }\end{array}$ & $\begin{array}{c}\text { Confidence } \\
(\mathbf{0} \div \mathbf{1})\end{array}$ & Efficiency [\%] \\
\hline$\# 1$ & 1206 & 0.56 & 94.16 \\
\hline$\# 2$ & 1257 & 0.65 & 84.17 \\
\hline$\# 3$ & 1192 & 0.60 & 90.83 \\
\hline$\# 4$ & 1301 & 0.54 & 80.00 \\
\hline$\# 5$ & 1251 & 0.58 & 98.33 \\
\hline Average & 1242 & 0.59 & 89.50 \\
\hline
\end{tabular}

Programming methods selection

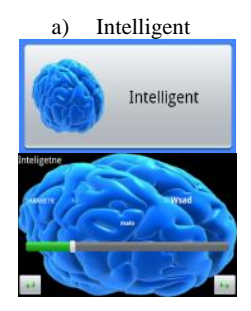

b) Classic
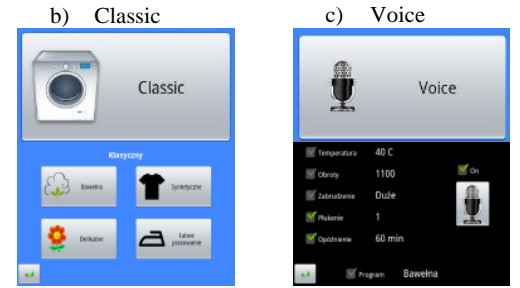

d)

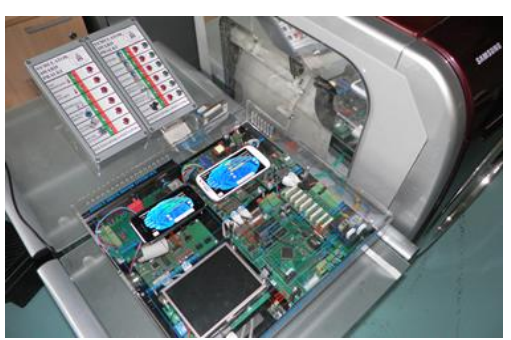

Fig. 6. View of the controller communication interfaces and view of the controller electronics

Finally, a special washing machine programming method, called "Intelligent", was proposed and implemented. It resembles the dialog between human and washing machine and is based on "Fuzzy Control" approach. During the programming phase the user has the possibility to set all parameters like: temperature, spin velocity, washing time, number of rinsing etc. in full range of washing machine possibilities. In the programming the graphical interface is used, which displays slider potentiometers on a screen. The user may move the slider, settling the washing parameters this way. All mentioned above programming methods are presented in Fig. 6. The software enables to switch intuitively and automatically between these three methods. For example, if the user starts to speak, the system is immediately going to voice recognition mode and so on. During the washing process its status is presented on a screen, and the user may stop the execution of the program, change some parameters and continue the washing process. The settled program and its parameters may be stored in the memory and induced again at any time. In Fig. 6d the view of the controller and smartphones during programming process are shown.

\section{SUMMARY}

The new 32-bit microcontroller designed and built at PUT was developed and implemented on the washing machine. Several prepared washing programs were tested, verified and approved. In the build controller both simple and advanced human-machine interfaces were developed. As a result the controller may be equipped with one, chosen for a client interface, like: typical interface with switches and LCD, mono or color touch-screen. Alternatively, the user may opt out of typical modules and may decide to use only smartphone or laptop for washing machine programming. Additionally the user may use the voice communication, programming and parameters setting. In the project also special intelligent methods of washing machine programming have been developed. All prepared methods were successfully tested. The proposed human washing machine interfaces may be implemented. The washing machines with such interfaces may be successfully offered on the market and find many clients.

\section{REFERENCES}

1. Anusuya M.A., Katti S.K. (2010), Speech recognition by machine, a review, ArXiv Prep,. ArXiv10012267.

2. Bascetta L., Rocco P., Zanchettin A.M., Magnani G. (2012), Velocity control of a washing machine: A mechatronic approach, Mechatronics, 22, 778-787.

3. Bourgeois J., van der Linden J., Kortuem G., Price B.A., Rimmer C. (2014), Conversations with my washing machine: an in-the-wild study of demand shifting with self-generated energy, ACM Press, 459-470.

4. Brown G. (2012), Discovering the STM32 Microcontroller, Indiana University

5. Chahuara P., Portet F., Vacher M. (2017), Context-aware decision making under uncertainty for voice-based control of smart home, Expert Syst. Appl., 75, 63-79.

6. Gundogdu K., Bayrakdar S., Yucedag I. (2017), Developing and modeling of voice control system for prosthetic robot arm in medical systems, J. King Saud Univ. - Comput. Inf. Sci.

7. Mallikarjun S. (2006), 32-bit MCUs offer high integration, high functionality, Electron. Prod., 49, 40-43.

8. Milecki A., Pittner G. (2015), Design of 32-bit washing machine controller, Solid State Phenomena, 220-221, 463-469.

9. Neto P., Pires J.N., Moreira A. (2010), High-level programming and control for industrial robotics: Using a hand-held accelerometerbased input device for gesture and posture recognition, Ind. Robot Int. J., 37, 137-147.

10. Norberto Pires J. (2005), Robot-by-voice: Experiments on commanding an industrial robot using the human voice, Ind. Robot Int. J., 32, 505-511.

11. Portet F., Vacher M., Golanski C., Roux C., Meillon B. (2013), Design and evaluation of a smart home voice interface for the elderly: acceptability and objection aspects, Pers. Ubiquitous Comput., 17, 127-144.

12. Risteska Stojkoska B.L., Trivodaliev K.V. (2017), A review of Internet of Things for smart home: Challenges and solutions, J. Clean. Prod., 140, 1454-1464.

13. Tsarouchi P., Athanasatos A., Makris S., Chatzigeorgiou X., Chryssolouris G. (2016a), High level robot programming using body and hand gestures, Procedia CIRP, 55, 1-5.

14. Tsarouchi P., Makris S., Chryssolouris G. (2016b), Human robot interaction review and challenges on task planning and programming, Int. J. Comput. Integr. Manuf., 29, 916-931.

Acknowledgements: The research work reported here was supported by the Polish Ministry of Science and Education grants no. 02/22/DSPB/1389. 